\title{
Aufsatz ÖR
}

\section{Prof. Dr. Wolfgang Kahl, M.A. und Wiss. Mitarb. James Bews \\ Rechtsfragen der Energiewende - Teil 1}

DOI 10.1515/jura-2014-0116

\section{A. Hintergrund}

Der Begriff der »Energiewende« bezeichnet nach Angaben des Bundesministeriums für Wirtschaft und Energie (BMWi) den »Weg in eine Zukunft ohne Atomenergie - hin $\mathrm{zu}$ einer Industriegesellschaft, die dem Gedanken der Nachhaltigkeit und der Verantwortung gegenüber kommenden Generationen verpflichtet ist. Dabei soll unser Strom bis $2035 \mathrm{zu} 55$ bis 60 Prozent und bis 2050 zu 80 Prozent aus erneuerbaren Energiequellen kommen. «1 Das Konzept, das die deutsche Energiepolitik der letzten 15 Jahre geprägt hat, ruht somit auf zwei Säulen: Dem Ausstieg aus der Kernenergie sowie dem Umstieg der Energieerzeugung auf Erneuerbare Energien (EE)².

Angesichts seiner Ubiquität ${ }^{3}$ kann die Erstarkung des Konzepts der »Energiewende« zu einem Rechtsbegriff ${ }^{4}$ nicht mehr bestritten werden. Mittlerweile handelt es sich sogar um einen Gesetzesbegriff 5 . In dem von dem Ausbau der Windenergie besonders betroffenen Schleswig-Holstein existiert ein »Ministerium für Energiewende, Landwirtschaft, Umwelt und ländliche Räume«.

Ihren ideengeschichtlichen Ursprung findet die Nutzung von EE, die auch dem wesentlich älteren Nachhaltigkeitsgedanken ${ }^{6}$ verhaftet ist, in den Überlegungen des britischen Philosophen und Ökonomen William Stanley

$1 \mathrm{http} / /$ www.bmwi.de/DE/Themen/Energie/Energiewende/energie wende-zum-erfolg-fuehren.html (25. 7. 2014).

2 So auch Heselhaus, EurUP 2013, 137 (137).

3 Eine im Juli 2014 unter dem Stichwort »Energiewende« durchgeführte Recherche in der Rechtsdatenbank juris ergab knapp 1300 Ergebnisse.

4 Vgl. hierzu Klement, Verantwortung, 2006, S. $34 \mathrm{ff}$.

5 Vgl. § 14 III 1 EnStatG oder Ziff. 682 02-330 des mittlerweile außer Kraft getretenen ERP-Wirtschaftsplangesetzes 2012.

$6 \mathrm{Zu}$ dessen Begriffsgeschichte Kahl, in: ders. (Hrsg.), Nachhaltigkeit als Verbundbegriff, 2008, S. 1 (6ff., 16f.).

Wolfgang Kahl, M.A.: Der Autor ist Professor für Öffentliches Recht und Europarecht an der Universität Heidelberg.

James Bews: Der Autor ist Wissenschaftlicher Mitarbeiter am Lehrstuhl von Prof. Kahl.
Jevons zu den Auswirkungen der Endlichkeit der KohleRessourcen auf die englische Industriegesellschaft ${ }^{7}$. In dem an fossilen Energieträgern relativ armen Deutschland gewann dieser Gedanke während des 1. Weltkriegs Aufwind, wo der "geschlossene Handelsstaat ${ }^{8}$ bereits im Jahr 1914 den planmäßigen Ausbau der Wasserkraft vorantrieb. Obwohl die nationalsozialistische Energiepolitik primär auf territoriale Expansion zur Sicherstellung ausreichender fossiler Ressourcen setzte, spielte auch hier die Autarkie verheißende Wasserkraft eine gewisse Rolle. Die Ölkrisen von 1973 und 1979 schließlich führten den westlichen Industriegesellschaften ihre Abhängigkeit von fossilen Energieträgern und die Notwendigkeit alternativer Energiequellen in erschreckender Deutlichkeit vor Augen.

Begriffsgeschichtlich hielt die »Energiewende« in ihrer modernen Gestalt (Atomausstieg und Ausbau der EE) wohl erstmals 1980 mit einer Veröffentlichung des Öko-Instituts ${ }^{9}$ Einzug in den deutschen Sprachraum und erreichte spätestens im Anschluss an die 2002 durch das Bundesministerium für Umwelt, Naturschutz, Bau und Reaktorsicherheit (BMU) veranstaltete Fachtagung »Energiewende - Atomausstieg und Klimaschutz« auch politische Beachtung.

Ungefähr auf das Jahr 2002 lässt sich auch der Umbruch in der deutschen Energiepolitik taxieren. Den Anfang machte die erste Rot-Grüne Bundesregierung unter Bundeskanzler Schröder (1998-2005), die sich in ihrem Koalitionsvertrag der Förderung von EE und dem Atomausstieg verpflichtete ${ }^{10}$. Das Kabinett Merkel I (2005-2009) führte dieses Konzept im Wesentlichen mit dem 2007 beschlossenen und im Folgejahr in verbindliches Recht umgesetzten »Integrierten Energie- und Klimaprogramm « ${ }^{11}$ fort. Eine - wenn auch vorübergehende - Kehrtwende erfolgte unter dem Kabinett Merkel II (2009-2013) durch das »Energiekonzept 2010«, das einen zeitlichen Aufschub

7 Jevons, The coal question, 1865.

8 Brandt, Deutsche Industrie im Kriege 1914/15, 1915, S. $85 \mathrm{f}$.

9 Krause/Bossel/Müller-Reißmann, Energiewende - Wachstum und Wohlstand ohne Erdöl und Uran, 1980.

10 Aufbruch und Erneuerung - Deutschlands Weg ins 21. Jahrhundert, 1998, S. $14 \mathrm{f}$

11 Bundesministerium für Umwelt, Naturschutz und Reaktorsicherheit, Das Integrierte Energie- und Klimaprogramm der Bundesregierung, 2007; vgl. dazu Bosecke, EurUP 2008, $122 \mathrm{ff}$. 
für den Atomausstieg vorsah ${ }^{12}$. Das als Reaktion auf die Havarie in Fukushima im Juni 2011 beschlossene Eckpunktepapier "Der Weg zur Energie der Zukunft - sicher, bezahlbar und umweltfreundlich « kehrte jedoch auf den eingeschlagenen Weg zurück und markiert die aktuellen Grundzüge ${ }^{13}$ der deutschen Energiepolitik.

Der nachfolgende, zweiteilige Beitrag gewährt einen Überblick über ausgewählte, besonders ausbildungs- und prüfungsrelevante grundsätzliche Rechtsfragen, die sich im Zusammenhang mit der Energiewende stellen und die, wie sich zeigen wird, keineswegs (nur) ein Thema für umwelt- oder energierechtliche Spezialisten sind. Vor dem Hintergrund der nationalen, europa- sowie völkerrechtlichen Rechtsgrundlagen (B.) wird der Atomausstieg (C.) sowie die europarechtliche Zulässigkeit der EE-Förderung (D.) beleuchtet, bevor auf die Grundlagen des Netzausbaus (E.) eingegangen wird. Abschließend werden unter F. die wesentlichen Ergebnisse festgehalten und der Blick auf die zukünftigen Herausforderungen gerichtet.

\section{B. Querschnittsmaterie}

Das Recht der Energiewende weist in zwei Richtungen einen Querschnittscharakter auf: In horizontaler, auf die nationale Ebene beschränkter, Perspektive bildet es die Schnittmenge unterschiedlicher verwaltungsrechtlicher Rechtsgebiete (I.). Der vertikale Querschnitt legt demgegenüber die Überwölbungen des »Energiewende-Rechts« offen, die sich aus dem (insbesondere internationalen und unionalen) rechtlichen Mehr-Ebenen-System ergeben (II.).

\section{Horizontale Betrachtung}

\section{Rechtsgrundlagen}

Das Energiewende-Recht lässt sich nicht einem einzelnen der bekannten Rechtsgebiete zuweisen. Vielmehr bestehen Querverbindungen zu so unterschiedlichen Gebieten wie dem Atom-, Umweltenergie-, Energiewirtschafts-, Klimaschutz-, Raumordnungs-, Bau-, Kommunal- und Steuerrecht.

12 Leitlinien für eine umweltschonende, zuverlässige und bezahlbare Energieversorgung, BT-Drs. 17/3049, S. 8f.

13 Die im Januar 2014 von der neuen Bundesregierung gefassten Kabinettsbeschlüsse von Meseberg enthalten zudem zahlreiche Änderungen der deutschen Energiepolitik, die über bloße Detailregelungen hinausreichen.
An erster Stelle ist die Atomausstiegsgesetzgebung zu nennen, die mehrere Änderungen des Atomgesetzes ${ }^{14}$ (AtG) bewirkt hat (dazu C.).

Besondere Bedeutung haben ferner die umweltenergierechtlichen Regelungen über die Förderung der Erzeugung von Strom sowie von Wärme und Kälte aus EE in dem Erneuerbare-Energien-Gesetz ${ }^{15}$ (EEG) (dazu D.) sowie dem Erneuerbare-Energien-Wärmegeset $z^{16}$.

Aber auch ohne ein wirksames Energieanlagenrecht wäre die Energiewende zum Scheitern verurteilt. Zu unterscheiden ist dabei zwischen dem auf Erzeugungsanlagen anwendbaren Recht, das sich im Wesentlichen im allgemeinen Bauplanungs- und Kommunalrecht findet und den auf Leitungsanlagen bezogenen Regelungen der §§ 12aff., 17aff. Energiewirtschaftsgesetz ${ }^{17}$ (EnWG), des Energieleitungsausbaugesetzes ${ }^{18}$ (EnLAG) und des Netzausbaubeschleunigungsgesetzes ${ }^{19}$ (NABEG) (s.u. E.).

Nicht Gegenstand des Rechts der Energiewende sind dagegen die Bestimmungen zur Steigerung der Energieeffizienz und zur Energieeinsparung ${ }^{20}$. Hierzu zählen das Kraft-Wärme-Kopplungsgesetz ${ }^{21}$, das Energieeinsparungsgesetz $^{22}$, das Energieverbrauchsrelevante-Produkte-Geset $^{23}$ sowie das Energieverbrauchskennzeichnungsgesetz $^{24}$. Auch das Treibhausgas-Emissionshandelsgesetz

14 Gesetz über die friedliche Verwendung der Kernenergie und den Schutz gegen ihre Gefahren v. 23. 12. 1959 i.d.F. v. 15. 7. 1985 (BGBl. I S. 1565), zuletzt geändert durch Gesetz v. 28. 8. 2013 (BGBl. I S. 3313). 15 Gesetz für den Ausbau erneuerbarer Energien v. 21.7. 2014 (BGBl. IS. 1066).

16 Gesetz zur Förderung Erneuerbarer Energien im Wärmebereich v. 7. 8. 2008 (BGBl. I S. 1658), zuletzt geändert durch Gesetz v. 21. 7. 2014 (BGBl. I S. 1066).

17 Gesetz über die Elektrizitäts- und Gasversorgung v. 7.7. 2005 (BGBl. I S. 1970), zuletzt geändert durch Gesetz v. 21. 7. 2014 (BGBl. I S. 1066).

18 Gesetz zum Ausbau von Energieleitungen v. 21. 8. 2009 (BGBl. I S. 2870), zuletzt geändert durch Gesetz v. 23. 7. 2013 (BGBl. I S. 2543). 19 Netzausbaubeschleunigungsgesetz Übertragungsnetz v. 28.7. 2011 (BGBl. I S. 1690), zuletzt geändert durch Gesetz v. 20. 12. 2012 (BGBl. I S. 2730).

20 Anders Jacobs, RELP 2012, 223 (226ff.).

21 Gesetz für die Erhaltung, die Modernisierung und den Ausbau der Kraft-Wärme-Kopplung v. 19. 3. 2002 (BGBl. I S. 1092), zuletzt geändert durch Gesetz v. 21. 7. 2014 (BGBl. IS. 1066).

22 Gesetz zur Einsparung von Energie in Gebäuden v. 1. 9. 2005 (BGBl. I S. 2684), zuletzt geändert durch Gesetz v. 4. 7. 2013 (BGBl. I S. 2197).

23 Gesetz über die umweltgerechte Gestaltung energieverbauchsrelevanter Produkte v. 27. 2. 2008 (BGBl. I S. 258), zuletzt geändert durch Gesetz v. 31. 5. 2013 (BGBl. I S. 1388).

24 Gesetz zur Kennzeichnung von energieverbrauchsrelevanten Produkten, Kraftfahrzeugen und Reifen mit Angaben über den Verbrauch an Energie und an anderen wichtigen Ressourcen v. 10. 5. 2012 (BGBl. I S. 1070). 
$\left(\right.$ TEHG) ${ }^{25}$ ist kein Energiewende-Recht. Während das TEHG dem Klimaschutzrecht zuzuordnen ist, liegen die Energieeffizienz- und Energieeinsparungsgesetze auf dem Grenzbereich von Klimaschutz- und Umweltenergierecht.

Seine aktuelle Gestalt verdankt das EnergiewendeRecht im Wesentlichen dem im Anschluss an die Reaktorkatastrophe von Fukushima und in Ausführung des Eckpunktepapiers »Der Weg zur Energie der Zukunft« 2011 beschlossenen Gesetzespaket, das in zahlreichen Bereichen erhebliche Veränderungen mit sich brachte ohne eine völlige Neugestaltung zu bewirken.

\section{Energiewende-Recht: Ein eigenständiges Rechtsgebiet?}

Zwar lässt sich das Energiewende-Recht trotz bestehender Überschneidungen vom Klimaschutz- und Umweltenergierecht $^{26}$ abgrenzen. Während auf der einen Seite das Klimaschutzrecht sich auf die »Verhinderung einer gefährlichen anthropogenen Störung des Klimasystems « ${ }^{27}$ beschränkt, bezieht das Energiewende-Recht auch den Ausstieg aus der (für sich genommen klimaneutralen) Atomenergie in seinen Zielkatalog ein. Auf der anderen Seite umfasst das Umweltenergierecht die Energieeinsparungs- und Energieeffizienzgesetze, während es die Ausstiegsgesetzgebung und das Recht des Netzausbaus nicht erfasst.

Der Annahme eines eigenen Rechtsgebiets »Energiewende-Recht« steht aber vor allem die Prozesshaftigkeit seines Regelungsgegenstands entgegen. Schon der Wortsinn des Begriffs der Energiewende verweist auf den vorübergehenden Charakter des erfassten Realphänomens. Sind der Atomausstieg sowie die Umstellung der Energieerzeugung auf EE erst einmal erreicht, würde sich ein auf diese Ziele ausgerichtetes Rechtsgebiet von selbst auflösen. Zwar ist auch anderen Rechtsgebieten ein solches ephemeres Element nicht völlig fremd ${ }^{28}$. Angesichts der Vorhersehbarkeit der Zielerreichung verlöre jedoch die systematisierende Wirkung der Bildung von Rechtsgebieten ihren Eigenwert.

25 Gesetz über den Handel mit Berechtigungen zur Emission von Treibhausgasen v. 21. 7. 2011 (BGBl. I S. 1475), zuletzt geändert durch Gesetz v. 15. 7. 2013 (BGBl. I S. 2431).

26 Vgl. zu diesen Gärditz, JuS 2008, 324 (324f., 329).

27 Schmidt/Kahl/Gärditz, Umweltrecht, 9. Aufl. 2014, § 6 Rn. 5.

28 Für das Regulierungsrecht etwa Fetzer, Staat und Wettbewerb in dynamischen Märkten, 2013, S. 18, 170.

\section{Vertikale Betrachtung}

Das auf die Energiewende bezogene Recht erweist sich auch im Vertikalverhältnis als mehrschichtig.

\section{Bundes-, Landes- und Kommunalrecht}

Das nationale Energiewende-Recht kennt neben den dargestellten bundesgesetzlichen Regelungen Vorschriften auf kommunaler Ebene sowie auf Ebene des Landesrechts. Kommunalrechtlich sind insbesondere die Satzungen zur Steuerung der Errichtung von EE-Erzeugungsanlagen zu nennen. Landesrechtlich ist auf die in einigen Bundesländern existierenden Klimaschutzgesetze ${ }^{29}$ hinzuweisen.

\section{Europarecht}

Das Energierecht der EU hat insgesamt eine erhebliche, wenn auch wechselhafte Determinationskraft für die deutsche Energiewende entfaltet. Die energierechtliche Kompetenzgrundlage des Art. 194 I AEUV erklärt neben dem in lit. a genannten Ziel der Sicherstellung eines Energiebinnenmarkts in lit. c u.a. die »Förderung der Energieeffizienz und von Energieeinsparungen sowie [die] Entwicklung neuer und erneuerbarer Energiequellen« zu den Zielen der europäischen Energiepolitik.

Während das Augenmerk der EU-Energiepolitik zunächst auf die Schaffung eines Energiebinnenmarkts gerichtet $w^{30}{ }^{30}$, ist in den letzten Jahren eine Schwerpunktverlagerung in Richtung des Energieumweltrechts $\mathrm{zu}$ beobachten. In der Mitteilung »Eine Energiepolitik für Europa $«^{31}$ aus dem Jahr 2007 wurde ein EE-Anteil an dem Gesamtstromverbrauch von 20\% angestrebt. Dieses Ziel wurde durch das Klimaschutzpaket von 2009 bestätigt und durch das Ziel der Reduktion der $\mathrm{CO}_{2}$-Emissionen um 20\% gegenüber 1990 ergänzt ( $20 \%$ und $20 \%$ bis 2020 $)^{32}$. Auch in der Kommissionsmitteilung »Energie 2020 - Eine Strategie für wettbewerbsfähige, nachhaltige und sichere Energie « ${ }^{33}$ wird das 2009 formulierte $20 \%$ und 20\%-Ziel bestätigt, wobei dem Bereich der Energieeffizienz besondere Bedeu-

29 Vgl. etwa KSG BW und KSG NRW.

30 Durch drei Richtlinienpakete aus den Jahren 1996/98, 2003 und 2009 wurde eine zunehmende Vertiefung des Energiebinnenmarkts erreicht.

31 Mitteilung der Kommission v. 10. 1. 2007 (KOM [2007] 1 endg.). 32 Schlussfolgerungen des Vorsitzes v. 13. 2. $2009(17271 / 1 / 08$ REV 1), Tz. $19 \mathrm{ff}$.

33 Mitteilung der Kommission v. 10. 11. 2010 (KOM [2010] 639 endg.). 
tung eingeräumt wird. In der Mitteilung »Erneuerbare Energien - ein wichtiger Akteur im europäischen Energiemarkt $\aleph^{34}$ von 2012 werden schließlich die Integration der EE in den Strommarkt sowie der Ausbau der Energieinfrastruktur ins Auge gefasst.

Das allgemeine Klimaschutz- und Umweltenergierecht wird auf europäischer Ebene durch die Rechtsakte auf dem Gebiet des Emissionshandels, der Energieeffizienz sowie des Rechtsrahmens für die Abscheidung und Speicherung von $\mathrm{CO}_{2}$ (CCS) konturiert. Energiewende-spezifische Regelungen ergeben sich daneben aus den EERichtlinien $^{35}$.

In den jüngsten Konsultationen zu einer EU-Energiepolitik für den Zeitraum von 2020 bis 2030 zeichnet sich eine Kehrtwende ab. Das aktuelle Grünbuch der Kommission »Ein Rahmen für die Klima- und Energiepolitik im Zeitraum 2020 bis 2030 « $^{36}$ rückt von der Vorgabe verbindlicher Ziele für den EE-Ausbau ab und nimmt primär die Reduktion der Treibhausgasemissionen in den Blick. Zwar kündigt sich (auch von Seiten der Unionsinstitutionen ${ }^{37}$ ) Widerstand gegen diesen Richtungswechsel an. Angesichts der erheblichen Divergenzen der nationalen Energieagenden - insbesondere bezüglich des Beitrags der Kernenergie zur $\mathrm{CO}_{2}$-Reduktion - ist das gewandelte Verständnis aber wohl Ausdruck der realpolitischen Notwendigkeit eines unionsweiten Kompromisses.

\section{Völkerrecht}

Wesentliche Impulse für den Wandel der deutschen Energiepolitik gingen schließlich von dem - zwischenzeitlich erlahmten - völkerrechtlichen Klimaschutzrecht aus. Die 1992 in Rio de Janeiro vereinbarte UN-Klimarahmenkonvention hat sich zum Ziel gesetzt, die Treibhausgaskonzentration in der Atmosphäre zu stabilisieren. Durch das 1997 beschlossene Kyoto-Protokoll ${ }^{38}$ wurden erstmals verbindliche Klimaschutzziele für die Industriestaaten festgelegt. Deutschland verpflichtete sich, seinen $\mathrm{CO}_{2}$-Ausstoß bis 2012 gegenüber dem Wert von 1990 um 21\% zu reduzieren. Mangels Abschlusses eines auch die Entwicklungs- und Schwellenländer einbeziehenden umfassenden Post-Kyoto-Abkommens wurde in Doha 2012 eine nur für einige Staaten geltende Verlängerung des Kyoto-Protokolls beschlossen. Die Anstrengungen der UN-Klimarahmenkon-

34 Mitteilung der Kommission v. 6. 6. 2012 (KOM [2012] 271 endg.). 35 RL 2001/77/EG; RL 2009/28/EG.

36 Mitteilung der Kommission v. 22. 1. 2014 (KOM [2014] 15 endg.). 37 Vgl. dazu Lehmann/Gawel/Strunz, ZUR 2014, $193 \mathrm{f}$.

38 BGBl. 2002 II S. 966. vention wurden durch die Vereinbarungen auf den G8Gipfeln in L'Aquila (2009) und Camp David (2012) flankiert, bei denen sich auch einige wichtige Schwellenländer erstmals zu dem auf der UN-Konferenz in Cancún (2010) vereinbarten Zwei-Grad-Ziel bekannten. Auf der für 2015 geplanten Klimakonferenz in Paris soll schließlich ein neues Abkommen mit für alle Mitgliedstaaten verbindlichen Zielen abgeschlossen werden.

Der bisherige völkerrechtliche Rahmen stellt dabei ausschließlich auf den übergeordneten Aspekt des Klimaschutzes durch Reduktion des $\mathrm{CO}_{2}$-Ausstoßes ab. Vorgaben hinsichtlich der zur Erreichung dieses Ziels gewählten Mittel ergeben sich hieraus nicht. Die Vertragsstaaten haben insofern die Wahl zwischen dem EE-Ausbau, der Nutzung von Atomenergie sowie Energieeffizienz- und Energieeinsparmaßnahmen.

\section{Atomausstieg}

Der Ausstieg aus der Nutzung der Kernenergie zur gewerblichen Erzeugung von Energie wird als »Herzstück der Energiewende ${ }^{39}$ bezeichnet. Auf der Grundlage des AtG wurden - mit Hilfe erheblicher staatlicher Fördergelder seit 1957 in Deutschland insgesamt 110 kerntechnische Anlagen betrieben. Der mit dem Schlagwort "Atomausstieg " paraphrasierte Wandel der deutschen Atompolitik vollzog sich in vier Stufen, die jeweils eigene staatsrechtliche und verwaltungsrechtliche Probleme aufwerfen.

\section{Ausstiegsgesetz 2002}

Fall 1: Die deutschen Energieversorgungsunternehmen (EVU) A, B, C und D, die die Rechtsform einer AG haben und in Deutschland neben anderen Energieerzeugungsanlagen auch Kernkraftwerke betreiben, sind der Auffassung, das Ausstiegsgesetz, das die Betriebsgenehmigungen von Kernkraftwerken mit der Erzeugung einer bestimmten Elektrizitätsmenge (Reststrommenge) wirkungslos werden lässt, verletze sie in ihren Grundrechten. Trifft dies zu, wenn die Anteile der C zu 90\% in der Hand des Bundeslands X, die Anteile der D vollständig in der Hand des ausländischen Staats S, der Mitglied der Europäischen Union ist, sind?

Die Rot-Grüne Bundesregierung traf 2000 mit den betroffenen EVU eine Vereinbarung (sog. Atomkonsens I), in dem der sukzessive Ausstieg aus der Kernenergie vereinbart wurde. In Umsetzung dieser Vereinbarung beschloss der

39 Degenhart, DVBl. 2013, 207 (207). 
Bundestag 2002 das sog. Ausstiegsgesetz ${ }^{40}$. Dieses fügte dem Genehmigungserfordernis für den Betrieb von Kernkraftwerken aus $\S 7$ I 1 AtG einen Satz 2 an, der die Erteilung neuer Betriebsgenehmigungen verbietet. Außerdem wurde $\S 7$ Ia AtG eingefügt, der bestimmt, dass bestehende Genehmigungen ablaufen, sobald die betreffende Anlage eine bestimmte Menge an Elektrizität erzeugt hat (Reststrommenge). Die Reststrommengen wurden dabei so kalkuliert, dass die Amortisation der Investitionen für jedes Kraftwerk sowie die Erwirtschaftung eines angemessenen Gewinns durch eine voraussichtliche Gesamtlaufzeit von 32 Jahren sichergestellt sind.

Das Ausstiegsgesetz wirft insbesondere ${ }^{41}$ grundrechtliche Rechtsfragen auf. Problematisch ist neben der Grundrechtsfähigkeit der EVU (1.) die Verletzung von Art. 14 I GG (2.) und Art. 12 I GG (3.).

\section{Grundrechtsfähigkeit der EVU}

Als Betreiber von Atomkraftwerken sind die vier großen deutschen EVU E.ON, RWE, Vattenfall und EnBW betroffen. Ihre Grundrechtsfähigkeit bemisst sich nach Art. 19 III GG.

E.ON, RWE und EnBW, die als AG organisiert sind, haben gemäß § 1 I 1 AktG eigene Rechtspersönlichkeit. Die Rechtsfähigkeit der Vattenfall $\mathrm{GmbH}$ ergibt sich aus $\S 13$ I 1 GmbHG. Damit sind sie als »juristische Personen« i.S.d. Art. 19 III GG anzusehen ${ }^{42}$. Auch einer Einordnung als »inländische« Vereinigung steht nichts entgegen.

Die Grundrechtsfähigkeit ist aber aus zwei Gründen problematisch. Zum einen hat das BVerfG juristischen Personen des Privatrechts vereinzelt die Grundrechtsfähigkeit mit der Begründung abgesprochen, dass sie eine öffentliche Aufgabe erfüllen ${ }^{43}$. Der Aufgabenkreis der Energieversorgung ist dem Bereich der Daseinsvorsorge zuzuordnen und somit als öffentliche Aufgabe anzusehen ${ }^{44}$. Das unbestimmte Kriterium der »öffentlichen Aufgabe« rechtfertigt jedoch nicht die Aberkennung der Grundrechtsfähigkeit von $\mathrm{EVU}^{45}$.

\footnotetext{
40 Gesetz zur geordneten Beendigung der Kernenergienutzung zur gewerblichen Erzeugung von Energie v. 22. 4. 2002 (BGBl. I S. 1351). 41 Zur Problematik der "paktierten Gesetzgebung« vgl. Kloepfer/ Bruch, JZ 2011, 377 (380ff.).

42 Zum Begriff der juristischen Person in Art. 19 III GG vgl. Pieroth/ Schlink/Kingreen/Poscher, Grundrechte, 29. Aufl. 2013, Rn. 162.

43 So BVerfG, NJW 1987, 2501 (2502); NJW 1996, 584.

44 BVerfGE 38, 258 (270f.); Schmidt-Preuß, in: Isensee/Kirchhof (Hrsg.), HStR IV, 3. Aufl. 2006, § 93 Rn. 43.

45 Remmert, in: Maunz/Dürig, GG, 70. EL 2013, Art. 19 III Rn. 63 (2009). A. A. Winter, ZfU 2012, 209 (242).
}

Zum anderen ist die Grundrechtsfähigkeit von EnBW, RWE und Vattenfall wegen ihrer spezifischen Beteiligungsverhältnisse problematisch. Die Anteile der EnBW befinden sich zu 90\% in öffentlicher Hand. Bei RWE machen öffentliche Anteilseigner $25 \%$ aus. Vattenfall wird sogar zu 100\% vom schwedischen Staat beherrscht. Ist eine juristische Person des Privatrechts teilweise in öffentlichem Eigentum, spricht man von einem "gemischt-wirtschaftlichen« Unternehmen. Im Gegensatz zu staatlichen Eigengesellschaften, bei denen der Staat alle Anteile hält und deren Grundrechtsfähigkeit $\mathrm{zu}$ Recht abgelehnt wird $^{46}$, ist die Grundrechtsberechtigung gemischt-wirtschaftlicher Unternehmen umstritten. Die wohl h.L. verneint diese bei Vorliegen eines staatlichen Beherrschungsverhältnisses ${ }^{47}$, wobei im Einzelnen unklar bleibt, wann eine derartige Beherrschung anzunehmen ist ${ }^{48}$. Diese Auffassung verweist auf die Grundrechtsverpflichtung von durch die öffentliche Hand beherrschten gemischt-wirtschaftlichen Unternehmen ${ }^{49}$. Eine gleichzeitige Grundrechtsverpflichtung und Grundrechtsberechtigung sei nicht möglich (Konfusionsargument) ${ }^{50}$. Außerdem müsse die Möglichkeit einer staatlichen »Grundrechtserschleichung " ausgeschlossen werden. Auch das BVerfG tendiert zu dieser Auffassung ${ }^{51}$.

Allerdings ist eine schematische Anwendung der Konfusionsthese, die ihren gedanklichen Ursprung in der Ablehnung der Grundrechtsberechtigung öffentlich-rechtlicher juristischer Personen hat, nicht angebracht ${ }^{52}$. Rechtsstreitigkeiten zwischen öffentlich-rechtlichen Personen sind nämlich in aller Regel Kompetenzstreitigkeiten, für die das verfassungsrechtliche Organstreitverfahren (Art. 93 I Nr. $1 \mathrm{GG}$ ), die Kommunalverfassungsbeschwerde (Art. 93 I Nr. 4b GG) sowie verwaltungsgerichtliche Organstreitverfahren als vorrangige Rechtsbehelfe bereit stehen. Bei Streitigkeiten zwischen gemischt-wirtschaftlichen Unternehmen und Hoheitsträgern existiert dagegen kein der-

46 Manssen, Staatsrecht II, 10. Aufl. 2013, Rn. 84. 47 S. nur Badura, Staatsrecht, 5. Aufl. 2012, C Rn. 14; Dreier, in: ders. (Hrsg.), GG, Bd. I, 3. Aufl. 2013, Art. 19 III Rn. 78; Huber, in: v. Mangoldt/Klein/Starck (Hrsg.), GG, Bd. I, 6. Aufl. 2010, Art. 19 Rn. 289ff.; Kahl, in: ders./Waldhoff/Walter (Hrsg.), Bonner Kommentar zum GG, 167. EL. 2014, Art. 1 III Rn. 287 ff. (i.E.).

48 Vgl. dazu Selmer, in: Merten/Papier (Hrsg.), HGR II, 2006, § 53 Rn. $19 \mathrm{ff}$.

49 Vgl. dazu BVerfGE 128, 226. Falllösung hierzu Bews/Greve, JURA 2012, $723 \mathrm{ff}$

50 Vgl. allg. zum Konfusionsargument BVerfGE 21, 362 (369f.); Jarass, in: ders./Pieroth, GG, 13. Aufl. 2014, Art. 19 Rn. 23.

51 Zuletzt BVerfGE 115, 205 (237).

52 Gersdorf, Öffentliche Unternehmen im Spannungsfeld zwischen Demokratie- und Wirtschaftlichkeitsprinzip, 2000, S. 71f. 
artiges Konkurrenzverhältnis mehrerer Rechtsbehelfe, sodass der hinter der Konfusionsthese stehende Zweck nicht einschlägig ist. Außerdem zeigt die Anerkennung der Grundrechtsfähigkeit von Universitäten, Rundfunkanstalten und Kirchen, dass das Konfusionsargument nicht ausnahmslos gilt. Aus diesem Grund ist der Auffassung zu folgen, die unabhängig von dem Ausmaß der staatlichen Beteiligung eine Grundrechtsberechtigung gemischt-wirtschaftlicher Unternehmen annimmt ${ }^{53}$. Dem Grundrechtsschutz der privaten Anteilseigner der EnBW sowie des RWE ist insofern Vorrang vor der Gefahr staatlicher »Grundrechtserschleichung « einzuräumen ${ }^{54}$.

Anders stellt sich die Sachlage hinsichtlich der Vattenfall $\mathrm{GmbH}$ dar. Diese befindet sich zu 100\% im Eigentum des schwedischen Staats. Hier verfängt das Konfusionsargument mangels Grundrechtsbindung ausländischer Staaten (Art. 1 III GG) zwar schon im Ansatz nicht ${ }^{55}$. Dennoch ist eine Grundrechtsberechtigung abzulehnen ${ }^{56}$. Ausländische Staaten sind nämlich nicht grundrechtsberechtigt. Dies muss - vergleichbar zu der deutschen Hoheitsgewalt - unabhängig von ihrer Organisationsform gelten. Auch Art. 18 I AEUV ändert an diesem Ergebnis nichts. Der schwedische Staat kann sich nicht auf das allgemeine Diskriminierungsverbot berufen. Selbst wenn Art. 18 I AEUV aber in analoger Anwendung von Art. 54 II AEUV anwendbar wäre, läge hier keine Ungleichbehandlung vor, da auch eine Eigengesellschaft der Bundesrepublik Deutschland nicht grundrechtsberechtigt ist.

\section{Eigentumsfreiheit (Art. 14 I GG)}

Die EVU berufen sich bezüglich der Ausstiegsgesetzgebung auf ihre Eigentumsfreiheit aus Art. 14 I GG.

\section{a) Schutzbereich}

In sachlicher Hinsicht berechtigt Art. 14 I GG in seiner Gestalt als Bestandsgarantie den Grundrechtsträger einen

53 S. nur Bleckmann, Staatsrecht II, 4. Aufl. 1997, § 9 Rn. 12; Schmidt-Aßmann, BB 1990/Beil. 34, 1 (10ff.); Stern, Staatsrecht, Bd. III/1, 1988, S. 1170.

54 I. Erg. auch Di Fabio, Der Ausstieg aus der wirtschaftlichen Nutzung der Kernenergie, 1999, S. 84ff.; Kersten/Ingold, ZG 2011, 350 (352). A.A. Bruch/Greve, DÖV 2011, 794 (796); Kloepfer, DVBl. 2011, 1437 (1438f.); Kube, ZG 2000, 11 (17ff.); Schlömer, Der beschleunigte Ausstieg aus der friedlichen Nutzung der Kernenergie, 2013, S. 99f.; Wallrabenstein, HFR 2011, 108 (113).

55 Zutreffend Kloepfer, DVBl. 2011, 1437 (1439 Fn. 24).

56 So auch Bruch/Greve, DÖV 2011, 794 (796); Schlömer (Fn. 54), S. 98. Zweifelnd auch BVerfG, NVwZ 2010, 373 (374).
Eigentumsgegenstand zu erwerben, zu behalten, $\mathrm{zu}$ veräußern und zu nutzen ${ }^{57}$. Der verfassungsrechtliche Eigentumsbegriff ist dabei weiter auszulegen als das auf körperliche Sachen beschränkte zivilrechtliche Eigentum (§ 903 $\mathrm{BGB})^{58}$. Insbesondere erfasst Art. 14 I GG die Befugnis des Eigentümers, sein Eigentum nach seinem Willen, d.h. auch zur Erzeugung von Kernenergie, zu nutzen ${ }^{59}$. Der Umfang dieser Nutzungsbefugnis wird seinerseits einfachgesetzlich durch die erteilte atomrechtliche Genehmigung (§ 7 I AtG) konkretisiert (normgeprägter Schutzbereich) ${ }^{60}$. Diese Genehmigung erfährt für sich genommen zwar keinen Eigentumsschutz ${ }^{61}$. Allerdings erstreckt sich Art. 14 I GG auf die aus der Genehmigung fließende Rechtsposition in Gestalt der öffentlich-rechtlich konkretisierten Nutzungsbefugnis des Eigentümers ${ }^{62}$. Ob darüber hinaus auch der eingerichtete und ausgeübte Gewerbebetrieb dem Schutzbereich unterfällt, ist umstritten ${ }^{63}$, kann hier aber dahinstehen, da die Gesamtheit des Sacheigentums ohnehin von Art. 14 I GG erfasst ist, die darüber hinausgehenden immateriellen Werte (Kundenstamm, Marke, goodwill etc.) durch das Ausstiegsgesetz aber nicht berührt werden. Im Ergebnis können sich die EVU daher hinsichtlich der durch die atomrechtliche Genehmigung konkretisierten Nutzungsbefugnis an ihren Anlagen auf Art. 14 I GG berufen.

Für den Fall, dass auch der Schutzbereich des Art. 12 I GG im Hinblick auf die Nutzungsbefugnis der EVU an ihren Anlagen eröffnet ist (s.u. C.I.3.), besteht Idealkonkurrenz zu Art. 14 I GG. Eine Spezialität des Art. 12 I GG ist abzulehnen ${ }^{64}$.

57 Manssen (Fn. 46), Rn. 675.

58 Lege, JURA 2011, 507 (509).

59 Depenheuer, in: Merten/Papier (Hrsg.), HGR V, 2013, § 111 Rn. 56. 60 Ossenbühl, Verfassungsrechtliche Fragen eines beschleunigten Ausstiegs aus der Kernenergie, 2012, S. 21.

$61 \mathrm{Vgl}$. für die h.M. nur Depenheuer, in: v. Mangoldt/Klein/Starck (Fn. 47), Art. 14 Rn. 134; Jarass (Fn. 50), Art. 14 Rn. 13; Kube, ZG 2000, 11 (25f.); Ossenbühl, AöR 124 (1999), 1 (8). A. A. s. nur Schröder, in: FS Papier, 2013, S. 605 ff.; Stüer/Loges, NVwZ 2000, 9 (12).

62 Denninger, Verfassungsrechtliche Fragen des Ausstiegs aus der Nutzung der Kernenergie zur Stromerzeugung, 2000, S. 47 ff.; Kersten/Ingold, ZG 2011, 350 (355); Ossenbühl, AöR 124 (1999), 1 (9).

63 Dafür nur Papier, in: Maunz/Dürig (Fn. 45), Art. 14 Rn. $95 \mathrm{ff}$. (2010). Dagegen Wieland, EnWZ 2013, 252 (256). Das BVerfG (E 66, 116 [145]; 68, 193 [222f.]; NJW 2010, 3501 [3502]) hat die Frage bislang offen gelassen.

64 Vgl. nur Papier (Fn. 63), Art. 14 Rn. 222. A.A. Wallrabenstein, HFR 2011, 108 (115); Wieland, EnWZ 2013, 252 (253); Ziehm, ZNER 2012, 221 (223). 


\section{b) Eingriff}

Ein Eingriff in Art. 14 I GG liegt vor, wenn eine schutzfähige Position entzogen oder ihre Nutzung, Verfügung oder Verwertung beschränkt wird ${ }^{65}$. Dabei unterscheidet man mit Blick auf die unterschiedlichen Rechtfertigungsvoraussetzungen zwischen Enteignungen i.S.d. Art. 14 III GG und Inhalts- und Schrankenbestimmungen gemäß Art. 14 I 2 GG. Eine Enteignung liegt bei jeder rechtsförmlichen, individuell-konkreten, vollständigen oder teilweisen Entziehung eines Eigentumsgegenstands zur Erfüllung einer öffentlichen Aufgabe vor ${ }^{66}$. Alle sonstigen Beeinträchtigungen des einfachgesetzlich zugeordneten Eigentums sind Inhalts- oder Schrankenbestimmungen ${ }^{67}$. Nach diesen Grundsätzen stellt das Ausstiegsgesetz mangels Güterbeschaffungsvorgangs ${ }^{68}$ keine Enteignung dar. Die Beschränkung der aus der Genehmigung fließenden Nutzungsbefugnis begründet keinen Vorteil für die öffentliche Hand, den diese zur Erfüllung öffentlicher Aufgaben nutzen will. Vielmehr wird der Umfang der Nutzungsbefugnis durch das Ausstiegsgesetz abstrakt-generell neu bestimmt, sodass eine Inhalts- und Schrankenbestimmung anzunehmen ist ${ }^{69}$.

\section{c) Rechtfertigung}

Inhalts- und Schrankenbestimmungen müssen sich am Übermaßverbot messen lassen, d.h. sie bedürfen eines legitimen Zwecks zu dessen Erreichung sie geeignet, erforderlich und angemessen sind ${ }^{70}$. Der mit dem Ausstiegsgesetz verfolgte Zweck besteht in der geordneten Beendigung der gewerblichen Nutzung der Kernenergie und dem Schutz vor von der Atomenergie ausgehenden Gefahren für Leben, Gesundheit und Sachgüter (§ $1 \mathrm{Nr} .1$ und $2 \mathrm{AtG}$ ), dessen Legitimität nicht anzuzweifeln ist. Zur Erreichung dieses Zwecks ist das Verbot der Erteilung neuer Betriebs-

65 Manssen (Fn. 46), Rn. 686.

66 Lege, JURA 2011, 507 (513f.).

67 Hufen, Staatsrecht II, 4. Aufl. 2014, § 38 Rn. 28.

$68 \mathrm{Ob}$ die Enteignung begrifflich einen Güterbeschaffungsvorgang voraussetzt, ist umstritten; vgl. bejahend nur BVerfGE 104, 1 (10); Jarass (Fn. 50), Art. 14 Rn. 72; ablehnend dagegen Papier (Fn. 63), Art. 14 Rn. 524.

69 So auch Denninger (Fn. 62), S. 51ff.; Koch, NJW 2000, 1529 (1530ff.); v. Komorowski, JURA 2001, 17 (19); Langenfeld, DÖV 2000, 929 (932ff.). In Bezug auf die insoweit vergleichbare 13. AtG-Novelle Bruch/Greve, DÖV 2011, 794 (797); Ewer, NVwZ 2011, 1035 (1037); Kersten/Ingold, ZG 2011, 350 (357 f.); Kloepfer, DVBl. 2011, 1437 (1439). A. A. Di Fabio (Fn. 54), S. 127 ff.; Ossenbühl, AöR 124 (1999), 1 (9ff.); Schmidt-Preuß, NJW 2000, 1524 (1525f.); Wagner, NVwZ 2001, 1089, (1095f.); Wendt, in: Sachs (Hrsg.), GG, 6. Aufl. 2011, Art. 14 Rn. 157b. 70 Hufen (Fn. 67), § 38 Rn. 42. genehmigungen sowie die in der Zuteilung von Reststrommengen liegende Beschränkung erteilter Genehmigungen geeignet, da dies zu einer sukzessiven Beendigung des Betriebs von Kernkraftwerken und den damit verbundenen Risiken führt ${ }^{71}$. Die Regelung ist auch erforderlich, da kein gleich geeignetes, aber weniger intensives Mittel zur Erreichung des gesetzgeberischen Ziels ersichtlich ist. Insbesondere wäre der Erlass von Auflagen zur weiteren Verbesserung der technischen Betriebssicherheit der Kernkraftwerke weniger geeignet als eine vollständige Stilllegung. Fraglich ist alleine, ob die mit dem Ausstiegsgesetz verbundenen Beeinträchtigungen des Eigentums der Anlagenbetreiber nicht außer Verhältnis zu dem mit dem Ausstiegsgesetz verfolgten Zweck stehen.

Durch die Einführung der Reststrommengen wird die aus der ohne Beschränkung erteilten Betriebsgenehmigung fließende Nutzungsbefugnis der Anlagenbetreiber schwerwiegend beeinträchtigt. Mit Verbrauch der Reststrommengen erlöschen die Betriebsgenehmigungen ex lege. Ob diese Beeinträchtigung aufgrund der Sozialbindung der Kernkraftwerke (Art. 14 II GG) weniger schwer wiegt $^{72}$, kann an dieser Stelle offen bleiben ${ }^{73}$. In jedem Fall kommt der Gesetzgeber mit Erlass des Ausstiegsgesetzes seiner aus Art. 2 II 1 GG folgenden Schutzpflicht nach, die neben der Abwehr von Gefahren auch die Risikovorsorge fordert $^{74}$. Zudem streitet für die Angemessenheit des Ausstiegsgesetzes der in Art. 20a GG verankerte Schutz der natürlichen Lebensgrundlagen, speziell das Vorsorgeprinzip und das Nachhaltigkeitsprinzip in seiner ökologischen Ausprägung.

Schließlich ist zu berücksichtigen, dass der Gesetzgeber eine Ausgleichsregelung getroffen hat. Die Verhältnismäßigkeit einer Inhalts- oder Schrankenbestimmung kann sich daraus ergeben, dass der Gesetzgeber einen finanziellen (sog. "ausgleichspflichtige Inhalts- und Schrankenbestimmung «) oder (vorrangig) sachlichen Ausgleich für den Rechtsverlust schafft ${ }^{75}$. Das Ausstiegsgesetz

71 Die Eignung kann nicht unter Hinweis auf die in einigen Nachbarstaaten weiterhin betriebenen Kernkraftwerke abgesprochen werden, vgl. Ossenbühl (Fn. 60), S. 65.

72 So Bruch/Greve, DÖV 2011, 794 (798).

73 Hiergegen spricht, dass die Sozialbindung - die bei Energieerzeugungsanlagen anzunehmen ist - die Ermöglichung der Nutzung des betroffenen Eigentumsgegenstands durch die Allgemeinheit bezweckt. Vorliegend geht es aber nicht um die Sicherstellung der aus dem Anlagenbetrieb fließenden Vorteile für die Allgemeinheit (etwa die Energieversorgungssicherheit), sondern um den umgekehrten Fall der Vermeidung von durch den Anlagenbetrieb drohenden Gefahren.

74 Schulze-Fielitz, in: Dreier (Fn. 47), Art. 2 II Rn. 80.

75 Depenheuer (Fn. 61), Art. 14 Rn. 236 ff. 
gewährleistet den den Anlagenbetreibern aus Art. 14 I GG zukommenden Vertrauensschut $\mathrm{z}^{76}$ in den Fortbestand ihrer Rechtsposition durch die Zuteilung der Reststrommengen. Hierin liegt eine zeitliche Übergangsregelung, die wegen ihrer zeitlichen Unbestimmtheit keine Befristung ${ }^{77}$, sondern eine auflösende Bedingung (vgl. § 36 II Nr. 2 VwVfG, § 158 II BGB) der Betriebsgenehmigungen darstellt. Diese Übergangsregelung ist auch angemessen, da sie eine Amortisation der getätigten Investitionen sowie einen angemessenen Gewinn sicherstell ${ }^{78}$.

\section{Berufsfreiheit (Art. 12 I GG)}

Auch eine Verletzung der Berufsfreiheit (Art. 12 I GG) ist nicht anzunehmen.

Der Schutzbereich des Art. 12 I GG erfasst jede Tätigkeit, die der dauerhaften Schaffung und Erhaltung einer Lebensgrundlage dient und nicht wegen ihrer Sozial- und Gemeinschaftsschädlichkeit von vornherein schlechthin nicht schützenswert ist. Art. 12 I GG ist entgegen seinem Wortlaut als einheitliches Grundrecht anzusehen, das umfassend Wahl, Ausübung und Aufgabe eines Berufs oder einer Ausbildung schützt ${ }^{79}$. Bei dem Betrieb eines Kernkraftwerks handelt es sich um eine von Art. 12 I GG erfasste Tätigkeit.

In der auflösenden Bedingung der Betriebsgenehmigungen durch Zuteilung der Reststrommengen liegt eine rechtsförmliche, finale, unmittelbare und imperative Beeinträchtigung i.S.d. klassischen Eingriffsbegriffs, sodass dahinstehen kann, ob der weitere moderne Eingriffsbegriff trotz des von dem BVerfG postulierten Erfordernisses einer berufsregelnden Tendenz auf Art. 12 I GG anwendbar ist ${ }^{80}$.

Dieser Eingriff ist aber gerechtfertigt. Trotz ihrer zu starren Schematik ist die von dem BVerfG entwickelte Drei-Stufen-Lehre ${ }^{81}$ - insbesondere in Prüfungsarbeiten weiterhin als Ausgangspunkt für eine differenzierte Bestimmung der Rechtfertigungsvoraussetzungen geeignet. Danach ist in einem ersten Schritt zu fragen, ob es sich bei

76 Art. 14 GG ist insofern gegenüber dem rechtsstaatlichen Vertrauensschutz - auf den sich die Vattenfall GmbH berufen kann vorrangig; Huber (Fn. 47), Art. 19 Rn. 228.

77 So aber Roßnagel/Hentschel, UPR 2011, 1 (3); Wieland, in: Dreier (Fn. 47), Art. 14 Rn. 150.

78 Ewer, NVwZ 2011, 1035 (1038); Kloepfer, DVBl. 2011, 1437 (1442); $v$. Komorowski, JURA 2001, 17 (20).

79 Kloepfer, Verfassungsrecht, Bd. II, 2010, § 70 Rn. 6.

80 Krit. hierzu Breuer, in: Isensee/Kirchhof (Hrsg.), HStR VIII, 3. Aufl. 2010, § 171 Rn. $44 \mathrm{f}$.

81 BVerfGE 7, 377 (405ff.); krit. Pieroth/Schlink/Kingreen/Poscher (Fn. 42), Rn. $926 \mathrm{ff}$. der Regelung um eine Beschränkung der Berufswahl oder der Berufsausübung handelt. Eine Berufswahlregelung kommt dabei nur in Betracht, wenn die betroffene Tätigkeit ein eigenständiges Berufsbild darstellt ${ }^{82}$. Der Betrieb eines Kernkraftwerks weist eine solche Eigenständigkeit nicht auf. Dies ergibt sich daraus, dass die vier EVU sich nicht auf den Betrieb von Kernkraftwerken konzentrieren, sondern ihr Tätigkeitsfeld auch andere Energieerzeugungsarten einbezieht. Abzustellen ist daher nicht auf den Beruf des »Kernkraftwerksbetreibers«, sondern auf den des »Kraftwerksbetreibers « ${ }^{83}$, sodass eine Berufsausübungsregelung anzunehmen ist, die vorliegend durch »vernünftige Erwägungen des Gemeinwohls « ${ }^{84}$ gerechtfertigt ist. Hinsichtlich der weiteren Verhältnismäßigkeitsprüfung (Geeignetheit, Erforderlichkeit, Angemessenheit) ist auf das oben (zu Art. 14 I GG) Gesagte zu verweisen.

Lösung zu Fall 1: Eine Grundrechtsverletzung der D scheidet schon aufgrund ihrer vollständigen Beherrschung durch den ausländischen Staat S aus. Eigengesellschaften ausländischer Staaten sind nach Art. 19 III GG nicht grundrechtsfähig. Die Grundrechtsberechtigung der C scheitert dagegen nicht an der 90\%-Beteiligung der öffentlichen Hand. Gemischt-wirtschaftliche Unternehmen sind trotz ihrer Grundrechtsverpflichtung (Art. 1 III GG) grundrechtsberechtigt. Allerdings werden A, B und C nicht in ihren Grundrechten aus Art. 14 I GG und Art. 12 I GG verletzt. Die durch die atomrechtliche Genehmigung konkretisierte Nutzungsbefugnis der EVU an den in ihrem Eigentum stehenden Anlagen erfährt durch das Ausstiegsgesetz zwar eine Beschränkung in Form einer Inhalts- und Schrankenbestimmung (Art. 14 I 2 GG). Diese erfolgt aber im Interesse der aus Art. 2 II 1 GG folgenden staatlichen Schutzpflicht bzw. des im Staatsziel Umweltschutz (Art. 20a GG) verankerten Vorsorgeund ökologischen Nachhaltigkeitsprinzips und ist angesichts der in der Zuteilung der Reststrommengen liegenden Übergangsregelung in Gestalt auflösender Bedingungen der Betriebsgenehmigungen verhältnismäßig. Aus diesem Grund ist auch die in dem Ausstiegsgesetz liegende Berufsausübungsregelung (Art. 12 I GG) gerechtfertigt. Die durch Art. 2 II GG gewährleistete allgemeine Handlungsfreiheit tritt im Wege der Subsidiarität zurück.

\section{Laufzeitverlängerung}

Fall 2: Das Bundesland E ist der Auffassung, die Verlängerung der Laufzeiten der Kernkraftwerke durch die 11. AtG-Novelle, die eine Erhöhung der den Kernkraftwerken zugeteilten Reststrom-

82 BVerfGE 77, 84 (105ff.).

83 So auch Ewer, NVwZ 2011, 1035 (1036); Kersten/Ingold, ZG 2011, 350 (367); Kloepfer, DVBl. 2011, 1437 (1443); Wallrabenstein, HFR 2011, 108 (118); Wieland, EnWZ 2013, 252 (254). A.A. Di Fabio (Fn. 54), S. 102ff.; Ossenbühl (Fn. 60), S. 79.

84 BVerfGE 7, 377 (405). 
mengen um 1804,278 TWh bewirkt, sei verfassungswidrig, da das Gesetz ohne Zustimmung des Bundesrats erlassen worden sei. Hat eine zulässige Normenkontrolle vor dem BVerfG Aussicht auf Erfolg?

Nachdem die aus CDU/CSU und FDP bestehende neue Regierungskoalition 2009 in ihrem Koalitionsvertrag eine Verlängerung der Laufzeiten der deutschen Kernkraftwerke, die als »Brückentechnologie« auf dem Weg zur vollständigen Umstellung auf EE notwendig seien, vereinbart hatte ${ }^{85}$, beschloss der Bundestag die 11. AtG-Novelle ${ }^{86}$, die im Dezember 2010 in Kraft trat. Eine Zustimmung des Bundesrats wurde nicht eingeholt, da dort wegen der Abwahl der CDU/FDP-Landesregierung in Nordrhein-Westfalen keine Mehrheit für die Laufzeitverlängerung zu erwarten war. Durch die 11. AtG-Novelle wurde das Verbot der Neuerteilung von Betriebsgenehmigungen zwar aufrechterhalten. Gleichzeitig wurden die den Kraftwerken zugeteilten Reststrommengen aber um insgesamt 1804,278 TWh erhöht. Dies entsprach einer durchschnittlichen Laufzeitverlängerung von zwölf Jahren je Kraftwerk. Hiergegen legten einige Landesregierungen beim BVerfG einen abstrakten Normenkontrollantrag mit der Begründung ein, die 11. AtG-Novelle sei mangels erforderlicher Zustimmung des Bundesrats verfassungswidrig.

Ein zustimmungsbedürftiges Gesetz kommt nach Art. 78 2. Var GG nur bei Zustimmung des Bundesrats zu Stande. Die fehlende Zustimmung hat die Unwirksamkeit des Gesetzes zur Folge. Ein Gesetz ist aber nur dann zustimmungsbedürftig, wenn das Grundgesetz dies ausdrücklich vorsieht (Enumerationsprinzip) ${ }^{87}$.

Die Zustimmungsbedürftigkeit der 11. AtG-Novelle wurde primär ${ }^{88}$ unter Verweis auf Art. 87 c GG begründet. Danach können Gesetze, die aufgrund des Art. 73 I Nr. 14 GG (Erzeugung und Nutzung der Kernenergie zu friedlichen Zwecken) ergehen, mit Zustimmung des Bundesrats vorsehen, dass sie durch die Länder in Bundesauftragsverwaltung ausgeführt werden. Hintergrund dieser Regelung ist die Systementscheidung des Grundgesetzes für einen grundsätzlich in eigener Verantwortung liegenden Vollzug der Bundesgesetze durch die Bundesländer (Art. 83 GG).

85 Vgl. S. 29 des Koalitionsvertrags 2009 »Wachstum. Bildung. Zusammenhalt «.

86 11. Gesetz zur Änderung des Atomgesetzes v. 8. 12. 2010 (BGBl. I S. 1814).

87 Dietlein, in: Epping/Hillgruber (Hrsg.), BeckOK GG, 20. Ed. 2014, Art. 77 Rn. 20.

88 Daneben wird vereinzelt (Gaßner/Kendzia, ZUR 2010, 456 [459f.]) vertreten, die 11. AtG-Novelle sei wegen Art. 74 II GG zustimmungsbedürftig. Dagegen zu Recht de Witt, RdE 2010, 357 (361f.); Roßnagel/ Hentschel, UPR 2011, 1 (2); Scholz, NVwZ 2010, 1385 (1389f.).
Durchbrechungen dieses Regel-Ausnahme-Verhältnisses werden aufgrund der im Vergleich zu Art. 84 GG intensiveren Ingerenzrechte des Bundes bei der Bundesauftragsverwaltung (Art. $85 \mathrm{GG}$ ) an die Zustimmung des Bundesrats geknüpft.

Das AtG ordnet in § 24 I 1 grundsätzlich den Landesvollzug im Auftrag des Bundes an und unterfällt damit dem Zustimmungsvorbehalt des Art. 87c GG. Allerdings begründet nicht jede Änderung eines zustimmungsbedürftigen Gesetzes ein erneutes Zustimmungserfordernis ${ }^{89}$. Vielmehr muss das Änderungsgesetz seinerseits zustimmungsbedürftige Regelungen enthalten oder den die $\mathrm{Zu}$ stimmungspflicht auslösenden Teil des Gesetzes in qualifizierter Weise berühren ${ }^{90}$. § $24 \mathrm{I} 1 \mathrm{AtG}$ selbst wird durch die 11. AtG-Novelle nicht unmittelbar geändert. Nach der Judikatur des BVerfG löst eine bloß mittelbare Betroffenheit zustimmungsbedürftiger Teile des Gesetzes in zwei Fällen das Zustimmungserfordernis des Änderungsgesetzes aus.

Erstens ist ein Änderungsgesetz zustimmungspflichtig, wenn die Änderung materiell-rechtlicher Vorschriften eine wesentliche Änderung der Bedeutung und Tragweite der von den Ländern auszuführenden Verwaltungsaufgabe hervorruft ${ }^{91}$. Dem Bund soll verwehrt werden, ohne Einverständnis der Länder durch die Hintertür eine erhebliche Änderung des Gegenstands herbeizuführen, auf den sich die ursprüngliche Zustimmung bezog. Hierfür ist aber eine qualitative, d.h. inhaltliche Änderung der Vollzugstätigkeit erforderlich. Eine rein quantitative Erhöhung der Vollzugslast begründet auch angesichts des Ausgleichsanspruchs aus Art. 104a II GG kein erneutes Zustimmungsbedürfnis. Die 11. AtG-Novelle führt zu keiner Veränderung der materiellen Rechtsgrundlagen des AtG. Durch Erhöhung der Reststrommengen erfahren die Betriebsgenehmigungen lediglich eine zeitliche Verlängerung, was $\mathrm{zu}$ einer quantitativen Erhöhung der Verwaltungstätigkeit führt.

Zweitens ist eine Zustimmung des Bundesrats erforderlich, wenn ein befristetes zustimmungsbedürftiges Gesetz durch ein Änderungsgesetz verlängert wird ${ }^{92}$. In Bezug auf die 11. AtG-Novelle wurde argumentiert, durch das Ausstiegsgesetz seien die Betriebsgenehmigungen unter einen zeitlichen Vorbehalt gestellt worden, was einer Befristung des gesamten AtG gleichkomme. Durch Erhöhung der Reststrommengen durch die 11. AtG-Novelle werde das Au-

89 Kokott, in: Kahl/Waldhoff/Walter (Fn. 47), Art. 77 Rn. $85 \mathrm{ff}$. (2014).

90 Kersten, in: Maunz/Dürig (Fn. 45), Art. 77 Rn. 101 (2012).

91 BVerfGE 37, 363 (383), st. Rspr.

92 BVerfGE 8, 274 (295). 
Berkrafttreten der Genehmigungen um durchschnittlich zwölf Jahre hinausgeschoben. Dies sei einer Verlängerung der Geltungsdauer des AtG gleichzustellen. Gegen eine solche Konstruktion sprechen jedoch sowohl formelle wie auch inhaltliche Überlegungen. In formeller Hinsicht ist zu bedenken, dass nicht das AtG selbst, sondern lediglich die Genehmigungen durch das Ausstiegsgesetz unter einen zeitlichen Vorbehalt gestellt wurden. Außerdem liegt, wie oben ausgeführt, wegen der Unbestimmtheit des Zeitpunkts des Verbrauchs der Reststrommengen keine Befristung, sondern eine auflösende Bedingung vor. In materieller Hinsicht spricht die fehlende Vergleichbarkeit der Konstellationen gegen eine Übertragung dieser Rechtsprechung. Mit Ablauf eines befristeten Gesetzes endet die das Zustimmungserfordernis auslösende Vollzugstätigkeit vollumfänglich. Dieses Ergebnis wird durch die Verlängerung der Geltungsdauer des Gesetzes verhindert. Der durch das Ausstiegsgesetz frühere Ablauf der Betriebsgenehmigungen hätte die Bundesländer zwar von ihrer atomrechtlichen Betriebsüberwachungspflicht entbunden. Fortbestanden hätte aber die Landesaufsicht über die Stilllegung und den Abbau der Kraftwerke, die Aufsicht über die Entsorgung des Atommülls sowie die Aufgaben des Strahlenschutzes. Dieser Aufgabenkreis, der einen nicht nur unerheblichen Umfang hat, wird durch die 11. AtG-Novelle nicht berührt.

Lösung zu Fall 2: Der gemäß Art. 93 I Nr. 2 GG, § 13 Nr. 6 i.V.m. $\S \S 76 \mathrm{ff}$. BVerfGG zulässige Normenkontrollantrag des Bundeslands E gegen die 11. AtG-Novelle hat mangels Zustimmungsbedürfnis keine Aussicht auf Erfolg93. Eine Zustimmungspflicht aus Art. 87c GG ist abzulehnen. Weder wird die die Zustimmungspflicht auslösende Bestimmung des § 24 I 1 AtG unmittelbar geändert, noch liegt ein Fall qualifizierter mittelbarer Betroffenheit vor. Die 11. AtG-Novelle betrifft die Vollzugsaufgaben der Bundesländer nämlich nicht dergestalt, dass deren Bedeutung und Tragweite eine qualitative Änderung erfahren. Auch liegt kein der Verlängerung eines befristeten Gesetzes vergleichbarer Fall vor.

93 So auch Degenhart, AtW 2010, 684 (686ff.); de Witt, RdE 2010, 357 (359ff.); Kloepfer/Bruch, JZ 2011, 377 (378); Kotulla/Kilic, NVwZ 2010, 1449ff.; Kraß, in: FS Dolde, 2014, S. 223 (229); Moench/Ruttloff, DVBl. 2010, 865 (869ff.); Rebentisch, UPR 2010, 361 (363f.); Scholz, NVwZ 2010, 1385 (1388ff.); Schwarz, JZ 2010, 1118 (1120f.). A.A Däuper/Michaels/Ringwald, ZUR 2010, 451 (452ff.); Gaßner/Kendzia, ZUR 2010, 456 (459f.); Geulen/Klinger, NVwZ 2010, 1118ff.; Kendzia, DÖV 2010, 713 (715ff.); Papier, NVwZ 2010, 1113 (1115ff.); Reyes y Ráfaeles, Der Staat 53 (2013), 599 (609); Roßnagel/Hentschel, UPR 2011, 1ff.; Uerpmann-Wittzack, in: v. Münch/Kunig (Hrsg.), GG, Bd. 2, 6. Aufl. 2012, Art. 87 c Rn. 6; Wieland, ZNER 2010, $321 \mathrm{ff}$.

\section{III. "Moratorium«}

Fall 3: Der Betreiber des Kraftwerks K, das zu den ältesten Kernkraftwerken in Deutschland gehört, wird durch den Minister M des Bundeslands E aufgrund der Ereignisse in Fukushima aufgefordert, für einen Zeitraum von drei Monaten sein Kernkraftwerk stillzulegen. Zur Begründung wird ausgeführt, dass die schrecklichen Ereignisse eine Überprüfung der Sicherheitsstandards der deutschen Atomkraftwerke erforderlich machten. Dies gelte in besonderem Maße für ältere Kraftwerke wie das des K. Ist das »Moratorium« mit Blick auf $\S 19$ III 1, 3 AtG rechtmäßig?

Bearbeitervermerk: § 19 III AtG lautet auszugsweise: Satz 1: "Die Aufsichtsbehörde kann anordnen, dass ein Zustand beseitigt wird, der den Vorschriften dieses Gesetzes [...] widerspricht oder aus dem sich durch die Wirkung ionisierender Strahlen Gefahren für Leben, Gesundheit oder Sachgüter ergeben können.»

Satz 3: "Sie kann insbesondere anordnen, [...] Nr. 3: dass der Umgang mit radioaktiven Stoffen, die Errichtung und der Betrieb von Anlagen der in den $\S \S 7$ und 11 Abs. 1 Nr. 2 bezeichneten Art sowie der Umgang mit Anlagen, Geräten und Vorrichtungen der in $\S 11$ Abs. 1 Nr. 3 bezeichneten Art einstweilen oder, wenn eine erforderliche Genehmigung nicht erteilt oder rechtskräftig widerrufen ist, endgültig eingestellt wird.»

Am 11. März 2011 - also gut drei Monate nach Inkrafttreten der 11. AtG-Novelle - kam es aufgrund eines Seebebens zu einer Überschwemmung in dem japanischen Kernkraftwerk Fukushima, die in drei Reaktorblöcken zu einer Kernschmelze führte. Große Mengen radioaktiven Materials wurden freigesetzt und kontaminierten die Umgebung. 150000 Menschen mussten evakuiert werden; über 1500 Menschen starben. Diese katastrophalen Ereignisse führten dazu, dass die Stimmung in der deutschen Bevölkerung schlagartig zu Lasten der Kernenergie umschwang. Es kam zu »Anti-Atomkraft«-Demonstrationen in zahlreichen größeren deutschen Städten. Auch aus diesem Grund kündigten die Bundeskanzlerin und der Außenminister auf einer Pressekonferenz am 14. März 2011 eine Sicherheitsüberprüfung aller deutschen Kernkraftwerke und die Aussetzung der durch das 11. AtG bewirkten Laufzeitverlängerung an. Am darauffolgenden Tag wurde darüber hinaus die sofortige Abschaltung der sieben ältesten Kernkraftwerke angekündigt. Die zuständigen Minister der betroffenen Bundesländer forderten die Betreiber dieser Kraftwerke auf, ihre Anlagen für einen Zeitraum von drei Monaten stillzulegen. Als Rechtsgrundlage zogen sie $\S 19$ III 1 2. Alt. i.V.m. § 19 III 3 Nr. 3 2. Var. AtG heran. Zur Begründung verwiesen sie darauf, dass die Ereignisse in Japan den Verdacht begründeten, dass von den deutschen Kernkraftwerken Gefahren ausgingen. Dieser Verdacht könne nur durch entsprechende Sicherheitsüberprüfungen ausgeräumt werden. Bis zur Beendigung dieser 
Überprüfungen müssten besonders die ältesten Kraftwerke vom Netz bleiben.

Neben der Frage, ob die das »Moratorium « anordnenden Landesbehörden ihre Anhörungspflicht aus §28 I LVwVfG verletzt hatten ${ }^{94}$, wurde von den betroffenen Kraftwerksbetreibern das Vorliegen der materiellen Tatbestandsvoraussetzungen von § 19 III 12 . Alt. AtG bezweifelt. Dieser verlangt eine Gefahr für Leben, Gesundheit oder Sachgüter. Der allgemeine polizeirechtliche Gefahrenbegriff, der auch im Rahmen des § 19 III 1 2. Alt. AtG anwendbar ist ${ }^{95}$, liegt bei einer Sachlage vor, »bei der im Einzelfall die hinreichende Wahrscheinlichkeit besteht, dass in absehbarer Zeit ohne Eingreifen des Staats ein Schaden für die Schutzgüter der Norm verursacht wird « ${ }^{96}$. Dabei hängt die Größe der zu fordernden Wahrscheinlichkeit von dem Ausmaß des drohenden Schadens sowie der Schwere des jeweiligen Eingriffs $a b^{97}$. Auch im Rahmen des AtG muss im Hinblick auf die stillzulegende Anlage eine konkrete Gefahr vorliegen. Der Verweis auf abstrakte Gefahren der Kernenergie genügt hierfür nicht ${ }^{98}$. Eine solche konkret in den vom "Moratorium « betroffenen Anlagen liegende Gefahr war nicht ersichtlich. Alleine mit ihrem Alter lässt sie sich jedenfalls nicht begründen.

Zwar kann nach überwiegender Ansicht auch im Rahmen des $\S 19$ III 12. Alt. AtG auf die polizeirechtliche Figur des Gefahrenverdachts zurückgegriffen werden ${ }^{99}$. Dies führt jedoch zu keiner anderen Bewertung. Ein Gefahrenverdacht liegt nämlich nur vor, wenn der handelnde Beamte nach den konkreten Umständen von der Möglichkeit des Vorliegens einer konkreten Gefahr ausgehen konn$\mathrm{te}^{100}$. Spezielle Umstände, die einen solchen Verdacht begründeten, wurden aber nicht vorgetragen ${ }^{101}$.

Zu denken war jedoch an die Übertragung des in § 7 II Nr. 3 AtG geregelten Vorsorgeprinzips ${ }^{102}$ auf die Auslegung des Gefahrbegriffs in $\S 19$ III 1 2. Alt. AtG ${ }^{103}$. Das - u.a. auch aus $\S 5$ I Nr. 21 . Alt. BImSchG bekannte und verfas-

94 Hierzu BVerwG, ZUR 2014, 236ff.; VGH Kassel, ZUR 2013, 367 (368ff.).

95 Ossenbühl, DVBl. 1990, 598 (600f.).

96 Schoch, in: ders. (Hrsg.), Besonderes Verwaltungsrecht, 15. Aufl. 2013, Kap. 2 Rn. 133.

97 Pieroth/Schlink/Kniesel, Polizei- und Ordnungsrecht, 7. Aufl. 2012, $\S 4$ Rn. 7.

98 BVerwG, NVwZ 1989, 1170 (1170).

99 VGH Kassel, ZUR 2013, 367 (371).

100 Schoch (Fn. 96), Kap. 2 Rn. 145.

101 VGH Kassel, ZUR 2013, 367 (371f.).

$102 \mathrm{Zu}$ diesem allg. Schmidt/Kahl/Gärditz (Fn. 27), § 4 Rn. $20 \mathrm{ff}$.

103 Dafür Frenz, NVwZ 2011, 522 (525); Roßnagel, DÖV 1998, 1048 (1048ff.). A. A. Cosack/Enders, DVBl. 2011, 1446 (1448f.); Rebentisch, NVwZ 2011, 533 (534). sungsrechtlich (Art. 20a GG) fundierte - Vorsorgeprinzip wird angesichts der Gefahren der Kernenergie im Rahmen des AtG besonders streng ausgelegt und lässt lediglich ein nach dem Maßstab praktischer Vernunft in Kauf zu nehmendes Restrisiko $\mathrm{zu}^{104}$. Im Gegensatz zu dem allgemeinen Gefahrbegriff ist die Schwelle der Schadenswahrscheinlichkeit im Rahmen des Restrisikos niedriger anzusetzen. Gegen eine Übertragung dieses Maßstabs spricht aber der Wortlaut des $\S 19$ III 1 2. Alt. AtG, der im Gegensatz zu $\S 7$ II Nr. 3 AtG von einer »Gefahr « spricht. Auch der unterschiedliche Zweck der Vorschriften, der sich in der Gesetzessystematik widerspiegelt, verbietet die Gleichsetzung von Gefahr und Vorsorge. Während § 7 II Nr. 3 AtG nämlich schon im Rahmen des Genehmigungsverfahrens von der Kernenergie ausgehende abstrakte Gefahren verhindern soll, betrifft $\S 19$ III 12 2. Alt. AtG die nachträgliche Verhinderung von anlagenbezogenen konkreten Gefahren ${ }^{105}$. Schließlich würde durch eine Gleichsetzung von Gefahr und Vorsorgemaßstab der Anwendungsbereich des $\S 19$ III 11 . Alt. AtG entleert, der eine atomrechtliche Aufsichtsmaßnahme zulässt, wenn die Anlage gegen Vorschriften des AtG und damit auch gegen den in $\S 7$ II Nr. 3 AtG enthaltenen Vorsorgemaßstab verstößt. Auch in der Rechtsprechung des BVerwG wird die klare Trennung zwischen atomrechtlichem Genehmigungs- und Aufsichtsverfahren betont ${ }^{106}$.

Selbst wenn man jedoch die Übertragbarkeit des Vorsorgemaßstabs auf $\S 19$ III 1 2. Alt. AtG annähme, wären dessen Voraussetzungen nicht erfüllt ${ }^{107}$. Bei Erteilung der Betriebsgenehmigungen wurden keine von den Kraftwerken ausgehenden besonderen Risiken festgestellt. Aus dem Grundsatz des »dynamischen Grundrechtsschutzes" folgt zwar, dass Veränderungen des Stands von Wissenschaft und Technik auch nachträglich zur Anpassung der an das Kraftwerk zu stellenden Sicherheitsanforderungen führen können ${ }^{108}$. Hierzu gehören auch neue Erkenntnisse hinsichtlich der Beherrschbarkeit der Folgen eines Unfalls ${ }^{109}$. Derartige neue Erkenntnisse lagen aber nicht vor. Der pauschale Hinweis auf den Unfall in Fukushima und das Alter der Anlagen ist für sich genommen nicht ausreichend, um eine Anpassung der Sicherheitsanforderungen zu rechtfertigen.

104 Vgl. nur BVerfGE 49, 89 (143); BVerwGE 72, 300 (315f.).

105 Cosack/Enders, DVBl. 2011, 1446 (1448).

106 BVerwGE 104, 36 (47ff.).

107 VGH Kassel, ZUR 2013, 367 (372).

108 Vgl. dazu nur BVerfGE 49, 89 (137); BVerwGE 72, 300 (311f.).

109 VGH Kassel, ZUR 2013, 367 (372). 
Lösung zu Fall 3: Das von M verkündete »Moratorium« ist mangels Vorliegens einer Gefahr i.S.d. § 19 III 1 2. Alt. AtG rechtswidrig ${ }^{110}$. Der Gefahrbegriff erfordert eine in der konkreten Anlage begründete konkrete Wahrscheinlichkeit eines Schadens. Eine solche wurde von M nicht vorgetragen. Auch ein Gefahrenverdacht lag nicht vor, da dieser ebenfalls konkrete anlagenbezogene Anhaltspunkte für die Möglichkeit des Vorliegens einer Gefahr erfordert. Schließlich lägen selbst bei einer zweifelhaften Übertragung des Vorsorgemaßstabs aus § 7 II Nr. 3 AtG auf $\S 19$ III AtG dessen Voraussetzungen mangels konkreter Anhaltspunkte für ein von der Anlage des K ausgehendes Risiko nicht vor.

\section{Beschleunigter Ausstieg}

Fall 4: Der Kernkraftwerksbetreiber G ist der Auffassung, die 13. AtG-Novelle, die seine Betriebsgenehmigung zum 31. 12. 2017 befristet und die ihm durch die 11. AtG-Novelle zusätzlich zugeteilte Reststrommenge entzieht, verstoße gegen seine durch Art. 14 I GG verbriefte Eigentumsfreiheit. Er trägt vor, im Vertrauen auf die Laufzeitverlängerung vom Dezember $2010 \mathrm{im}$ Januar 2011 Investitionen in sein Kraftwerk getätigt zu haben, die nun wertlos geworden seien. Ist seine Rechtsauffassung zutreffend?

Nach Verkündung des "Moratoriums« entschloss sich die Bundesregierung, den endgültigen Atomausstieg voranzutreiben. Die Reaktorsicherheitskommission wurde damit beauftragt, die Sicherheit der in Betrieb befindlichen Kernkraftwerke zu untersuchen. Zudem sollte eine »Ethik-Kommission Sichere Energieversorgung " einen gesellschaftlichen Konsens durch Bewertung der ethischen und technischen Fragen der Atomenergie vorbereiten. Die Ethik-Kommission kam zu dem Ergebnis, ein Atomausstieg innerhalb eines Jahrzehnts sei möglich und erstrebenswert. Ein solcher spiegle auch den gesellschaftlichen Konsens wider, der trotz fehlender neuer Erkenntnisse zu einer Änderung der Wahrnehmung der von der Kernenergie ausgehenden Gefahren geführt habe ${ }^{111}$.

Daraufhin beschloss der Bundestag im Juni 2011 die 13. AtG-Novelle ${ }^{112}$, die einen Entzug der den Kernkraftwerken durch die 11. AtG-Novelle zusätzlich zugeteilten Reststrommengen anordnete. Zudem wurden in § 7 Ia AtG für die einzelnen Kraftwerke feste Fristen eingefügt, mit deren Ablauf die Betriebsgenehmigungen erlöschen. Die Fristen wurden so berechnet, dass den einzelnen Kraftwerken eine

110 So auch Cosack/Enders, DVBl. 2011, 1446 (1447ff.); Kloepfer/ Bruch, JZ 2011, 377 (386); Schwarz, BayVBl. 2013, 65 (67).

111 Ethik-Kommission Sichere Energieversorgung, Deutschlands Energiewende - Ein Gemeinschaftswerk für die Zukunft, 2011, S. $11 \mathrm{ff}$. 112 13. Gesetz zur Änderung des Atomgesetzes v. 31. 7. 2011 (BGBl. I S. 1704).
Laufzeit von 32 Jahren zusteht. Zur Begründung der 13. AtG-Novelle verwies der Gesetzgeber auf die von der Ethik-Kommission beschriebene geänderte Wahrnehmung der Risiken der friedlichen Nutzung der Kernenergie in der Bevölkerung.

Gegen die 13. AtG-Novelle wurden Verfassungsbeschwerden eingelegt, über die noch nicht entschieden ist ${ }^{113}$. In grundrechtlicher Hinsicht ${ }^{114}$ wird neben einer Beeinträchtigung der Berufsfreiheit (Art. 12 I GG), die im Vergleich zu dem Ausstiegsgesetz (s.o. C.I.3.) keine neuen Rechtsfragen aufwirft, insbesondere die Verletzung von Art. 14 I GG geltend gemacht.

\section{Schutzbereich}

Wie oben dargestellt, wird die durch die Anlagengenehmigung in ihrem Umfang definierte Nutzungsbefugnis der Kernkraftwerksbetreiber vom Schutzbereich des Art. 14 I GG erfasst. Im Hinblick auf die 13. AtG-Novelle von besonderem Interesse ist allerdings, ob auch die durch das Ausstiegsgesetz erstmals zugeteilten und durch die 11. AtGNovelle erhöhten Reststrommengen dem Art. 14 I GG zuzuordnen sind. Das ist umstritten ${ }^{115}$.

Das BVerfG geht in einem neueren Urteil wohl davon aus, dass die Reststrommengen dem Schutzbereich des Art. 14 I GG unterfallen ${ }^{116}$. Diese Ansicht ist jedoch abzulehnen. Öffentlich-rechtliche Rechtspositionen, wie die durch das AtG hoheitlich zugeteilten Reststrommengen, werden von Art. 14 I GG nur erfasst, wenn sie dem Rechtsträger »nach Art eines Ausschließlichkeitsrechts « zugeordnet sind und auf einer "nicht unerheblichen Eigenleistung « beruhen ${ }^{117}$. Zwar können die Kraftwerksbetreiber die ihnen zugeteilte Reststrommenge verbrauchen oder auf andere Kraftwerke übertragen, sodass eine ausschließliche Rechtszuordnung anzunehmen ist. Allerdings erfolgt

113 Az. 1 BvR 2821/11, 1 BvR 312/12, 1 BvR 1456/12 u.a.

114 Außerdem werden Verstöße gegen das Gesetzgebungsverfahren, die gesetzgeberische Begründungspflicht sowie Fehler bei der gesetzgeberischen Abwägungsentscheidung geltend gemacht; vgl. Degenhart, Gesetzgeberische Sorgfaltspflichten bei der Energiewende, 2013, S. 37 ff. Zur Frage der »Unumkehrbarkeit« des Atomausstiegs Ellerbrok, VR 2013, 148 (150ff.).

115 Für die Schutzbereichserstreckung sprechen sich aus: Bruch/ Greve, DÖV 2011, 794 (795); Degenhart, DVBl. 2013, 207 (210); Huber, DVBl. 2003, 157 (159f.); Kersten/Ingold, ZG 2011, 350 (371); Kloepfer, DVBl. 2011, 1437 (1438). A.A. Ekardt, NuR 2012, 813 (813); Ossenbühl (Fn. 60), S. $17 \mathrm{ff} . ;$ Wallrabenstein, HFR 2011, 108 (116); Wieland, EnWZ 2013, 252 (255f.); Ziehm, ZNER 2012, 221 (223).

116 BVerfG, NVwZ 2009, 921 (923).

117 BVerfGE 48, 403 (413); Depenheuer (Fn. 61), Art. 14 Rn. $69 \mathrm{ff}$. 
der Rechtserwerb nicht aufgrund einer Eigenleistung der Kraftwerksbetreiber ${ }^{118}$. Teilweise werden die Investitionen der Betreiber in die Kraftwerke als derartige Eigenleistung angesehen ${ }^{119}$. Das überzeugt jedoch nicht. Zum einen wurden die betreffenden Investitionen im Vertrauen auf die längeren Laufzeiten erst nach Zuteilung der zusätzlichen Reststrommengen getätigt. Würden solche nachträglichen Aufwendungen im Vertrauen auf den Fortbestand einer öffentlich-rechtlichen Begünstigung diese zu einer Eigentumsposition i.S.d. Art. 14 I GG erstarken lassen, hätte der Private die eigenhändige Ausweitung des Schutzbereichs in der Hand. Zum anderen soll das Kriterium der Eigenleistung die Zuordnung öffentlich-rechtlicher Rechtspositionen unter den Schutzbereich des Art. 14 I GG auf solche Fälle beschränken, in denen die öffentliche Hand eine Gegenleistung erhält. Nur in solchen Fällen nähert sich das öffentlich-rechtliche Rechtsverhältnis dem Zivilrechtsverkehr an. Die Investitionen in die Kraftwerke kommen aber nicht der öffentlichen Hand zu Gute, sondern dienen der Instandhaltung der Anlagen und verbleiben damit in der Sphäre des Kraftwerksbetreibers.

Gegen eine Schutzbereichserstreckung spricht zudem folgende Überlegung: Wie dargelegt, handelt es sich bei der Zuteilung der Reststrommengen um eine Übergangsregelung zur Vermeidung der Unverhältnismäßigkeit des Atomausstiegs (s.o. C.I.2.c). Die Betriebsgenehmigung wird unter die auflösende Bedingung des Verbrauchs der Reststrommenge gestellt. In dieser Gestalt konkretisiert die Genehmigung die Nutzungsbefugnis des Anlageneigentïmers. Da die Reststrommenge als auflösende Bedingung aber eine Einschränkung der zuvor durch eine zeitlich unbedingte Betriebsgenehmigung konkretisierten Nutzungsbefugnis darstellt, kann sie keine über diese Nutzungsbefugnis hinausgehende Eigentumsposition begründen.

Damit sind die Reststrommengen für sich genommen nicht Schutzbereichsgegenstand des Art. 14 I GG. Eigentumsrechtlicher Zuordnungsgegenstand ist vielmehr die Nutzungsbefugnis der Kraftwerksbetreiber in der Gestalt, die sie durch die auflösend bedingte Betriebsgenehmigung hat.

118 Krit. zur Übertragbarkeit des Eigenleistung-Kriteriums Schröder (Fn. 61), S. 611ff.

119 Kloepfer, DVBl. 2011, 1437 (1438).

\section{Eingriff}

Diese Rechtsposition wird in zwei Hinsichten beschränkt. Zum einen wird die auflösende Bedingung durch Entzug der durch die 11. AtG-Novelle zusätzlich zugesprochenen Reststrommengen $\mathrm{zu}$ Lasten der Anlagenbetreiber verschärft. Zum anderen werden die Betriebsgenehmigungen zusätzlich zu der auflösenden Bedingung unter eine Befristung gestellt. Bei diesen Maßnahmen handelt es sich um nachträgliche Verschärfungen der im Interesse der Anlagenbetreiber ergangenen Übergangsregelung und mithin um Inhalts- und Schrankenbestimmungen i.S.d. Art. 14 I 2 GG.

\section{Rechtfertigung}

Fraglich ist, ob der hierin liegende Eingriff verhältnismäßig ist. Der legitime Zweck liegt in der Reduzierung der Risiken der Nutzung der zivilen Kernenergie ${ }^{120}$. Im Gegensatz zu der Rechtslage bei dem »Moratorium « (s. o. C.III.) ist eine anlagenbezogene konkrete Gefahr entbehrlich. Unbeachtlich ist daher, dass die Havarie in Fukushima keine neuen Erkenntnisse über die Gefahren der Kernenergienutzung zu Tage gefördert hat. Auch eine bloße Änderung der Wahrnehmung einer potentiellen Gefahrenquelle ermöglicht dem Gesetzgeber im Rahmen des ihm zustehenden politischen Einschätzungsspielraums eine Änderung seiner bisherigen politischen Ausrichtung ${ }^{121}$.

Die Verschärfung der Übergangsregelung ist auch zur Erreichung dieses Zwecks geeignet und erforderlich. Problematisch ist allerdings ihre Angemessenheit. Wie gesehen (s.o. C.I.2.c), ist die Beeinträchtigung des Eigentums der Kraftwerksbetreiber mit den zu Gunsten des beschleunigten Atomausstiegs streitenden Art. 2 II 1, 20a GG in angemessenen Ausgleich zu bringen.

Bei der Prüfung der Angemessenheit ist insbesondere dem in Art. 14 I GG verkörperten Vertrauensschutz der Kraftwerksbetreiber in den Bestand ihres Eigentums Rechnung zu tragen. Ein reduzierter Vertrauensschutz ergibt sich nicht aus der Kürze des Zeitraums zwischen der 11. AtG-Novelle (Dezember 2010) und der 13. AtG-Novelle (Januar 2011) ${ }^{122}$ oder den (nicht durchgreifenden, s. o. C.II.) Zweifeln an der Verfassungsmäßigkeit der 11. AtG-Novel-

120 Vgl. BT-Drs. 17/6070, S. 1.

121 Bruch/Greve, DÖV 2011, 794 (797); Ewer, NVwZ 2011, 1035 (1036); Schröder, NVwZ 2013, 105 (108); Wollenteit, ZUR 2013, 323 (325f.). A. A. Degenhart, DVBl. 2013, 207 (209ff.).

122 So aber Bruch/Greve, DÖV 2011, 794 (798); Ewer, NVwZ 2011, 1035 (1038). 
$\mathrm{le}^{123}$. Vielmehr ist wegen der Außergewöhnlichkeit der nachträglichen Änderung einer Übergangsregelung von einer besonderen Schutzwürdigkeit des Vertrauens auszugehen $^{124}$. Fraglich ist daher, ob die Übergangsregelung in ihrer durch die 13. AtG-Novelle erhaltenen Gestalt dieses schutzwürdige Vertrauen hinreichend berücksichtigt. Dabei ist zwischen dem allgemeinen Vertrauen der Kraftwerksbetreiber in den Fortbestand ihrer Nutzungsbefugnis und dem besonderen Vertrauen in den Fortbestand der durch die 11. AtG-Novelle geschaffenen Übergangsregelung zu unterscheiden ${ }^{125}$.

Hinsichtlich des allgemeinen Vertrauenstatbestands hat die 13. AtG-Novelle die durch das Ausstiegsgesetz getroffene Übergangsregelung wieder in Kraft gesetzt und zusätzlich feste Fristen eingeführt, die den einzelnen Kraftwerken eine Laufzeit von 32 Jahren garantierten. Wie unter C.I.2.c dargestellt, genügt ein solcher Zeitrahmen für eine Amortisation der Investitionen sowie die Erzielung eines angemessenen Gewinns. Das allgemeine Vertrauen in den Fortbestand der Nutzungsbefugnis ist damit hinreichend geschützt.

123 Kersten/Ingold, ZG 2011, 350 (372f.).

124 In diese Richtung Schröder, NVwZ 2013, 105 (110). Eine - wenn auch nicht 1:1 übertragbare - Analogie kann zum aus befristeten Gesetzen folgenden Vertrauensschutz gezogen werden, der nach der Rspr. (BVerfGE 30, 392 [404]) nicht geringer ausfällt als bei unbefristeten Regelungen. Schulze-Fielitz, in: Hoffmann-Riem/SchmidtAßmann (Hrsg.), Innovation und Flexibilität des Verwaltungshandelns, 1994, S. 139 (159) geht sogar davon aus, dass für den befristeten Geltungszeitraum ein erhöhtes Vertrauen in den Fortbestand der Regelung anzunehmen ist.

125 In diese Richtung auch Bruch/Greve, DÖV 2011, 794 (798).
Problematisch ist allerdings, ob ein darüber hinausgehendes Vertrauen der Kraftwerksbetreiber in den Fortbestand der durch die 11. AtG-Novelle geänderten Übergangsregelung einen besonderen Ausgleich erfordert. In Betracht kommt eine finanzielle Ausgleichsregelung ${ }^{126}$, wenn die Betreiber im Vertrauen auf die verlängerte Laufzeitenregelung das normale Maß übersteigende Aufwendungen getätigt haben. Von einigen Kraftwerksbetreibern wurden solche zusätzlichen Investitionen behauptet ${ }^{127}$. Sollte es ihnen gelingen, diese Investitionen im Einzelnen darzulegen, wäre das Fehlen einer finanziellen Ausgleichsregelung als unverhältnismäßige Inhalts- und Schrankenbestimmung anzusehen ${ }^{128}$.

Lösung zu Fall 4: G wird durch die 13. AtG-Novelle in seiner Eigentumsfreiheit verletzt. Der Schutz des Art. 14 I GG erstreckt sich auf seine durch die Genehmigung konkretisierte Nutzungsbefugnis an den Anlagen; die ihm zugeteilte Reststrommenge selbst erfährt aber keinen Grundrechtsschutz. Die in der Befristung und Entziehung der Reststrommenge liegende Verschärfung der Übergangsregelung stellt eine Inhalts- und Schrankenbestimmung dar, die wegen des ersatzlosen Verlusts der im Vertrauen auf die Laufzeitenverlängerung vom Dezember 2010 von G im Januar 2011 getätigten Investitionen unangemessen und damit unverhältnismäßig ist.

126 Ein sachlicher Ausgleich scheidet bei nachträglicher Verschärfung einer Übergangsregelung denklogisch aus.

127 So soll die Vattenfall GmbH Investitionen in Höhe von 700 Mio. $€$ getätigt haben; Bruch/Greve, DÖV 2011, 794 (798).

128 So auch Bruch/Greve, DÖV 2011, 794 (799); Kersten/Ingold, ZG 2011, 350 (373f.); Kraß (Fn. 93), S. 244ff.; Schlömer (Fn. 54), S. $156 \mathrm{ff}$. A. A. Ewer, NVwZ 2011, 1035 (1037). 